\title{
Performance of Copeptin for Early Diagnosis of Acute Myocardial Infarction in an Emergency Department Setting
}

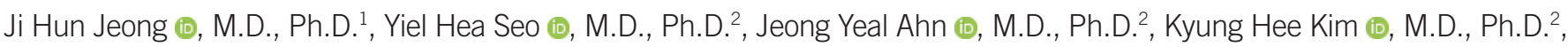

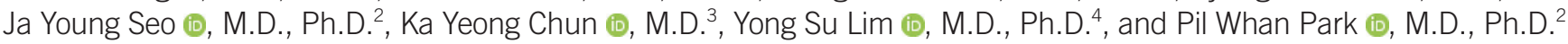

${ }^{1}$ Department of Laboratory Medicine, Chung Ang University Hospital, Seoul, Korea; ${ }^{2}$ Department of Laboratory Medicine, Gil Medical Center, Gachon University College of Medicine, Incheon, Korea; ${ }^{3}$ Department of Internal Medicine, Gimpo Woori Hospital, Gimpo, Korea; ${ }^{4}$ Department of Emergency Medicine, Gil Medical Center, Gachon University College of Medicine, Incheon, Korea

Background: Rapid and accurate diagnosis of acute myocardial infarction (AMI) is critical for initiating effective treatment and achieving better prognosis. We investigated the performance of copeptin for early diagnosis of AMI, in comparison with creatine kinase myocardial band (CK-MB) and troponin I (TnI).

Methods: We prospectively enrolled 271 patients presenting with chest pain (within six hours of onset), suggestive of acute coronary syndrome, at an emergency department (ED). Serum CK-MB, Tnl, and copeptin levels were measured. The diagnostic performance of CK-MB, Tnl, and copeptin, alone and in combination, for AMI was assessed by ROC curve analysis by comparing the area under the curve (AUC). Sensitivity, specificity, negative predictive value, and positive predictive value of each marker were obtained, and the characteristics of each marker were analyzed.

Results: The patients were diagnosed as having ST elevation myocardial infarction (STEMI; $\mathrm{N}=43$ ), non-ST elevation myocardial infarction (NSTEMI; $N=25)$, unstable angina $(N=78)$, or other diseases $(\mathrm{N}=125)$. AUC comparisons showed copeptin had significantly better diagnostic performance than $\mathrm{Tnl}$ in patients with chest pain within two hours of onset (AMI: $P=0.022, \leq 1$ hour; STEMI: $P=0.017, \leq 1$ hour and $P=0.010, \leq 2$ hours). In addition, $\mathrm{Tnl}$ and copeptin in combination exhibited significantly better diagnostic performance than CK-MB plus Tnl in AMI and STEMI patients.

Conclusions: The combination of Tnl and copeptin improves AMI diagnostic performance in patients with early-onset chest pain in an ED setting.

Key Words: Acute myocardial infarction, Copeptin, Troponin I, Creatine kinase myocardial band, Performance, ST elevation myocardial infarction, Non-ST elevation myocardial infarction
Received: April 19, 2019

Revision received: April 22, 2019

Accepted: August 7, 2019

\section{Corresponding author:}

Pil Whan Park, M.D., Ph.D.

Department of Laboratory Medicine, Gil Medical Center, Gachon University College of Medicine, 21 Namdong-daero 774beongil, Namdong-gu, Incheon 21565, Korea

Tel: +82-32-460-3834

Fax: +82-32-460-3415

E-mail: pwpark@gilhospital.com

\section{INTRODUCTION}

Chest pain is a common cause of emergency department (ED) visits and a major burden on healthcare resources [1]. Because not all patients need immediate medical treatment or interven- tion, ruling in and ruling out pathologies are equally important. Rapid diagnosis of acute myocardial infarction (AMI) is important for early initiation of appropriate treatment in patients with this life-threatening disorder; AMI must be excluded to ensure safe discharge of low-risk patients intended for outpatient follow- 
up. Biomarkers, such as creatine kinase myocardial band (CK$\mathrm{MB}$ ) and troponin I (Tnl), are considered the gold standards for the diagnosis of $\mathrm{AMI}$; however, their elevation requires some time once myocardial necrosis has occurred [2, 3]. Accordingly, early diagnosis of AMI in patients with no or mild Tnl elevation is challenging.

Biomarkers with a pathophysiological background independent of cell necrosis might facilitate early diagnosis of AMI. Copeptin, a 39-amino-acid peptide on the C-terminal portion of pro-arginine vasopressin, is an early biomarker and is secreted with vasopressin during acute events such as AMI [4]. By having a different pathophysiology with a time course that is complementary to that of $\mathrm{Tnl}$ as a biomarker of myocardial necrosis, copeptin may have value in the early diagnosis of AMI at initial presentation. Copeptin levels peak early ( $0-1$ hour) after AMI symptoms onset and decrease to routine levels within 12-36 hours in early presenters [5]. The Copeptin Helps in the Early Detection of Patients with Acute Myocardial Infarction (CHOPIN) trial demonstrated the value of copeptin in early rule-out of $\mathrm{MI}$ [6]. Furthermore, the 2015 European Society of Cardiology guidelines on the management of patients with acute coronary syndrome (ACS) presenting without ST-segment elevation myocardial infarction (STEMI) state that the use of copeptin with troponin may add diagnostic value [7]. However, recent studies have suggested that copeptin elevation in serum occurs under various conditions [8], such as lower respiratory tract infection [9], sepsis [10], and stroke [11], as well as after AMI [4, 12]; copeptin might be a useful non-specific biomarker [4]. We investigated the performance of copeptin to diagnose AMI as a way to overcome the delay in release of CK-MB and Tnl in patients with ACS in an ED setting.

\section{METHODS}

\section{Patient selection}

We enrolled 271 patients older than 18 years with chest pain onset within six hours of presentation and suspected ACS who presented at the ED of Gachon Gil Medical Center, Incheon, Korea, between May 2017 and August 2017. The time of chest pain onset was determined based on patient/bystander information, and the times of symptom onset and ED arrival were recorded in an electronic medical record. Patients with traumatic causes of chest pain were excluded. Initial patient assessments included a physical examination, 12-lead electrocardiogram (ECG), chest X-ray, and blood assays including conventional serum CK-MB and Tnl assays. The initial diagnosis was made by an emergency physician and verified by internal medicine physicians using all available data, which included serial ECG, cardiac markers, and percutaneous coronary intervention results. The clinical characteristics of all patients are summarized in Table 1. Their median age was 59.0 years, and $69.0 \%$ were men. Patients were classified into four groups by etiology: (1) ST elevation myocardial infarction (STEMI; $\mathrm{N}=43$ ), (2) non-ST elevation myocardial infarction (NSTEMI; $\mathrm{N}=25$ ), (3) unstable angina (UAP; $N=78$ ), and (4) other diseases (other; $N=125$ ). CK-MB, $\mathrm{Tnl}$, and copeptin levels were higher in patients with AMI than in patients with UAP or other diseases (Table 1). This study was approved by the Institutional Review Board of Gachon Gil Medical Center (GCIRB2017-160), which waived the requirement for informed consent.

Determination of serum CK-MB, Tnl, and copeptin levels Venous blood for investigational biomarker analysis was routinely collected at the time of ED arrival. Serum CK-MB and Tnl levels were measured using an automated, sandwich chemiluminescent immunoassay (Advia, Centaur XP, Siemens Healthcare Diagnostics Inc., Tarrytown, NY, USA). After routine laboratory tests, residual serum samples ( $\geq 500 \mu \mathrm{L}$ ) were kept frozen at $<-70^{\circ} \mathrm{C}$ until measurement of copeptin in August 2017. Morgenthaler, et al. [13] showed that prolonged frozen storage does not affect copeptin levels. Copeptin levels were measured using a commercial sandwich immunoluminometric assay, the BRAHMS Copeptin KRYPTOR kit on a BRAHMS KRYPTOR compact plus analyzer (BRAHMS GmbH, Hennigsdorf, Germany). The precision of the copeptin assay was tested at two levels ( $6.12 \mathrm{pmol} / \mathrm{L}$ and $110 \mathrm{pmol} / \mathrm{L}$ ) by running five replicates over five days. The within-run precision and between-run precision CVs were $<10 \%$, demonstrating satisfactory repeatability. The limit of quantification (LoQ) was $1.23 \mathrm{pmol} / \mathrm{L}$, and the analytical measurable range (AMR) was $500 \mathrm{pmol} / \mathrm{L}$. Initial studies used a copeptin cut-off level of 14 pmol/L; however, recent studies have suggested that a $10 \mathrm{pmol} / \mathrm{L}$ cut-off level might be more appropriate for ruling out early AMI [14, 15]. Accordingly, we used a second-generation copeptin assay and $10 \mathrm{pmol} / \mathrm{L}$ as the cut-off level.

\section{Statistical analysis}

Baseline characteristics including sex, age, and laboratory findings were analyzed using SPSS, Ver. 22.0 (IBM, Chicago, IL, USA). Data were not normally distributed. Hence, data were presented as median (interquartile range, IQR) and analyzed using the Kruskal-Wallis test. Pearson's chi-square test was used 


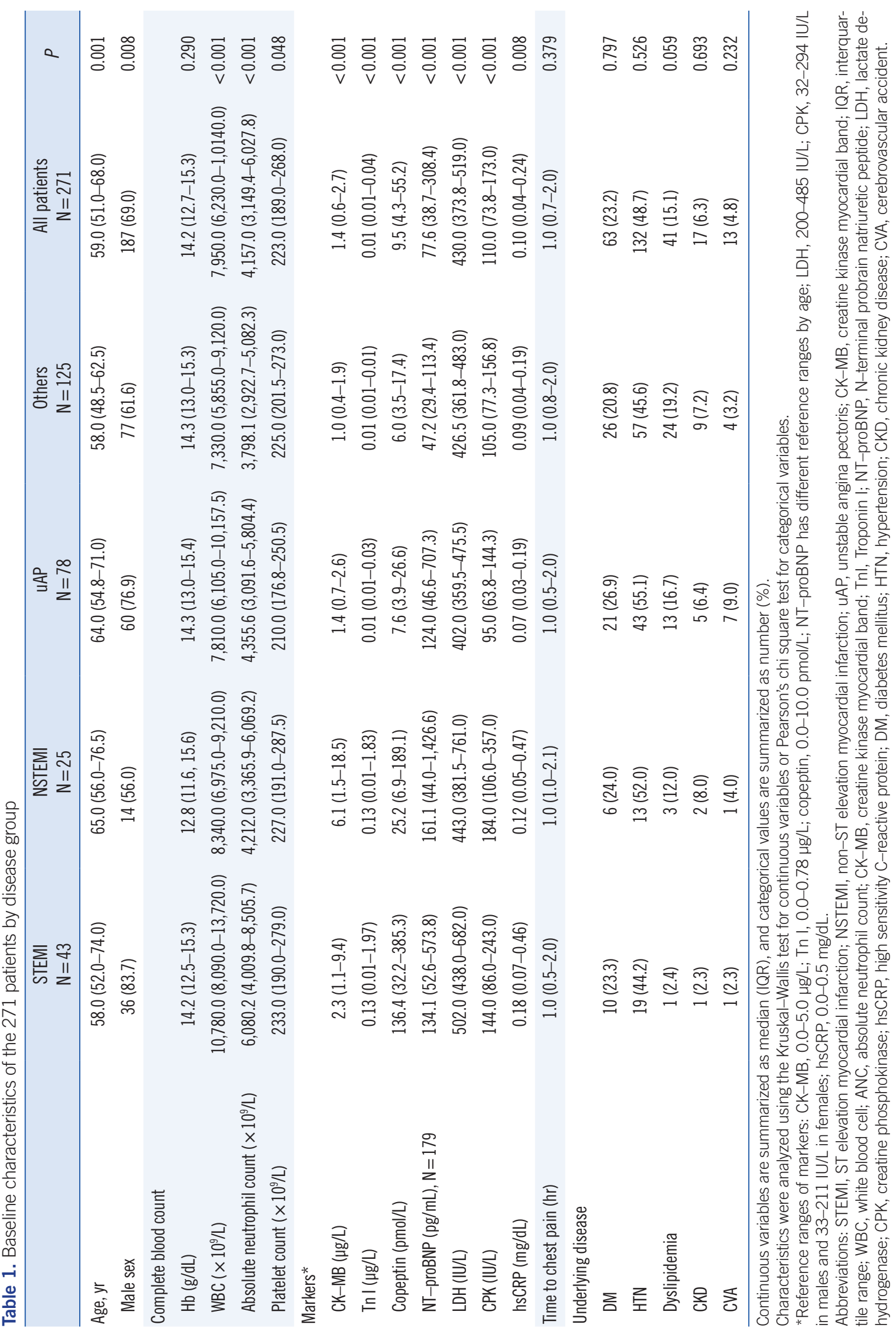


to compare categorical variables. $P<0.05$ was deemed significant.

Diagnostic performance was assessed by ROC curve analysis using MedCalc (Ver. 18.2.1; MedCalc Software, Ostend, Belgium), which was performed using Youden's index. This index is the point on the ROC curve furthest from the line of equality (diagonal line) and can be used to differentiate non-informative (area under the curve $[A \cup C]=0.5)$, less accurate $(0.5<A \cup C \leq$ $0.7)$, moderately accurate $(0.7<A \cup C \leq 0.9)$, highly accurate $(0.9<A \cup C<1)$, and perfect assays $(A \cup C=1)$. We assessed the performances of the three biomarkers alone and in combination by comparing AUCs [12]. ROC curve comparison to analyze the diagnostic performance of the three biomarkers was calculated for times of $\leq 1, \leq 2$, or $\leq 6$ hours after chest pain onset. The time of onset was defined as the time that had elapsed between symptom onset and ED presentation. Additionally, sensitivity, specificity, positive predictive values (PPVs), and negative predictive values (NPVs) for the markers were also assessed by applying a marker-specific cutoff value.

\section{RESULTS}

Diagnostic performance of markers

In AMI patients with a chest pain onset of $\leq$ one hour, the ROC AUC at ED arrival was 0.678 (95\% confidence interval [Cl], 0.5960.753) for CK-MB, 0.719 (95\% Cl, 0.639-0.790) for Tnl, and 0.855 (95\% Cl, 0.787-0.907) for copeptin. In AMI patients with a chest pain onset of $\leq$ two hours, the ROC AUC values for CKMB, Tnl, and copeptin were 0.693 (95\% Cl, 0.626-0.754), 0.750 (95\% Cl, 0.687-0.807), and 0.837 (95\% Cl, 0.780-0.884), re-

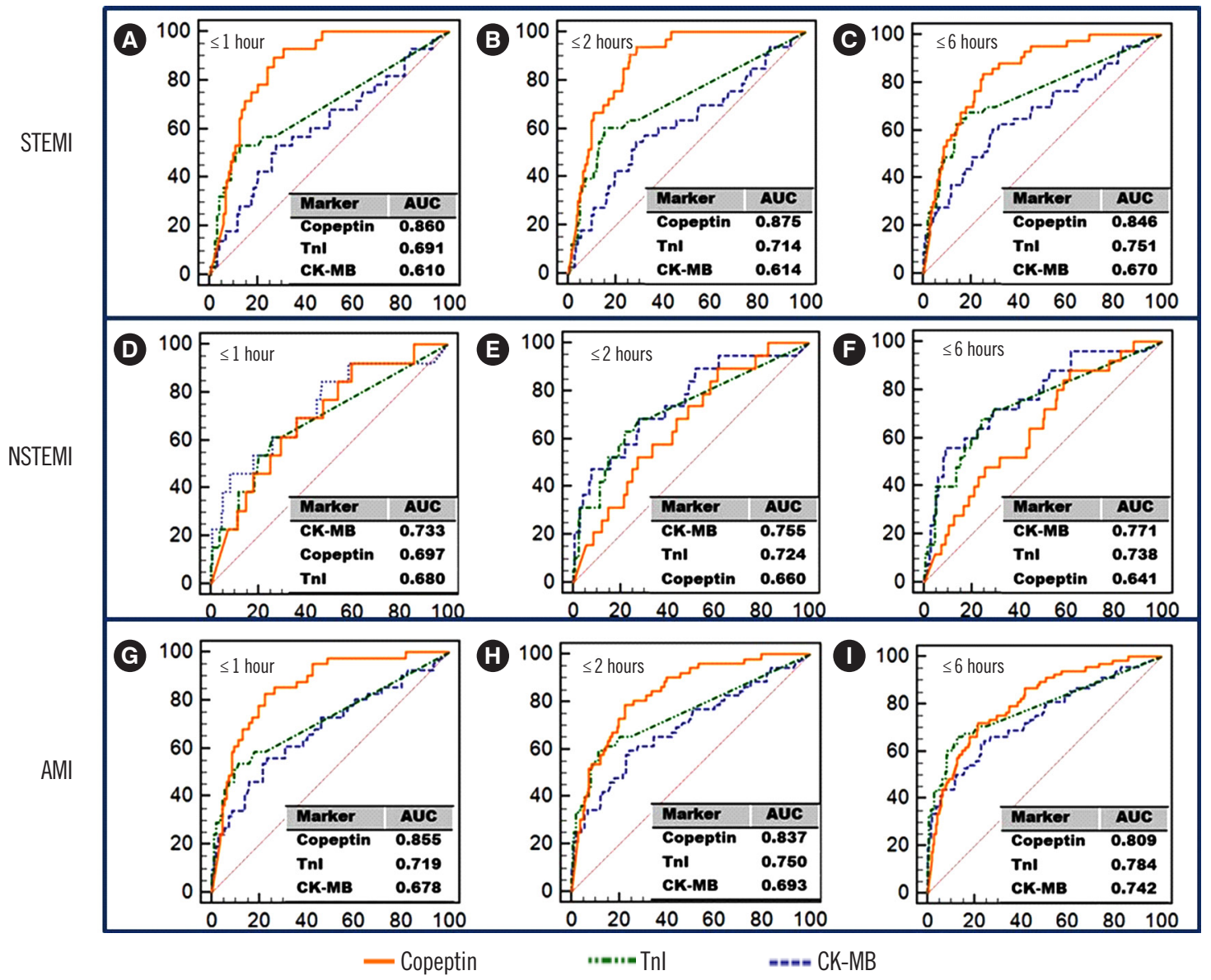

Fig. 1. ROC curves for CK-MB, Tnl, and copeptin according to the time of chest pain onset in AMI patients. The ROC curve plots sensitivity on the $Y$-axis and (1-specificity) on the $X$-axis. The area under the curve (AUC) of each marker is presented in each graph. Copeptin as a single marker was superior to CK-MB within six hours $[(A)-(C)]$ and to Tnl within two hours $[(A) \&(B)]$ in the STEMI group $(P<0.05)$. Copeptin showed better diagnostic performance than CK-MB within two hours $[(G) \&(H)]$ and Tnl within one hour in the $A M I$ group $(G)(P<0.05)$. Abbreviations: STEMI, ST elevation myocardial infarction; NSTEMI, non-ST elevation myocardial infarction; AMI, acute myocardial infarction; CK-MB, creatine kinase myocardial band; Tnl, troponin I. 
spectively. In AMI patients with a chest pain onset of $\leq$ six hours, the corresponding values were $0.742(95 \% \mathrm{Cl}, 0.685-0.793)$, 0.784 (95\% Cl, 0.731-0.832), and 0.809 (95\% Cl, 0.757-0.854), respectively. The diagnostic performance of CK-MB and TnI increased with time after chest pain onset. However, the AUC of copeptin peaked within two hours of pain onset (AMI: $\leq 1$ hour; STEMI: $\leq 2$ hours, and NSTEMI: $\leq 1$ hour). Furthermore, copeptin showed moderately accurate diagnostic performance in patients with AMI or STEMI (AUC: 0.809-0.875). ROC curve comparisons showed copeptin had significantly better diagnostic performance than $\mathrm{Tnl}$ in patients within 1-2 hours of chest pain onset (AMI: $P=0.022$ at $\leq 1$ hour after chest pain onset; STEMI: $P=0.017$ at $\leq 1$ hour, $P=0.010$ at $\leq 2$ hours).

Diagnostic performance of combined biomarkers

The use of copeptin as a single diagnostic biomarker or in com- bination with $\mathrm{Tnl}$ was superior to other markers or the CK-MB plus Tnl combination currently used for diagnosing AMI and STEMI (Figs. 1 and 2). ROC curve comparisons also showed that copeptin plus Tnl had significantly better diagnostic performance than CK-MB plus Tnl in the AMI and STEMI groups.

Analysis for sensitivity, specificity, PPV, and NPV

Tnl showed the highest specificity and PPV; however, NPV was the lowest among the three biomarkers. Copeptin presented the highest sensitivity and NPV but the lowest PPV among the biomarkers. The addition of copeptin to Tnl improved the sensitivity and NPV compared with Tnl alone (Table 2).

Biomarker response according to time after chest pain onset in AMI

Copeptin appeared to be able to diagnose AMI shortly after symp-

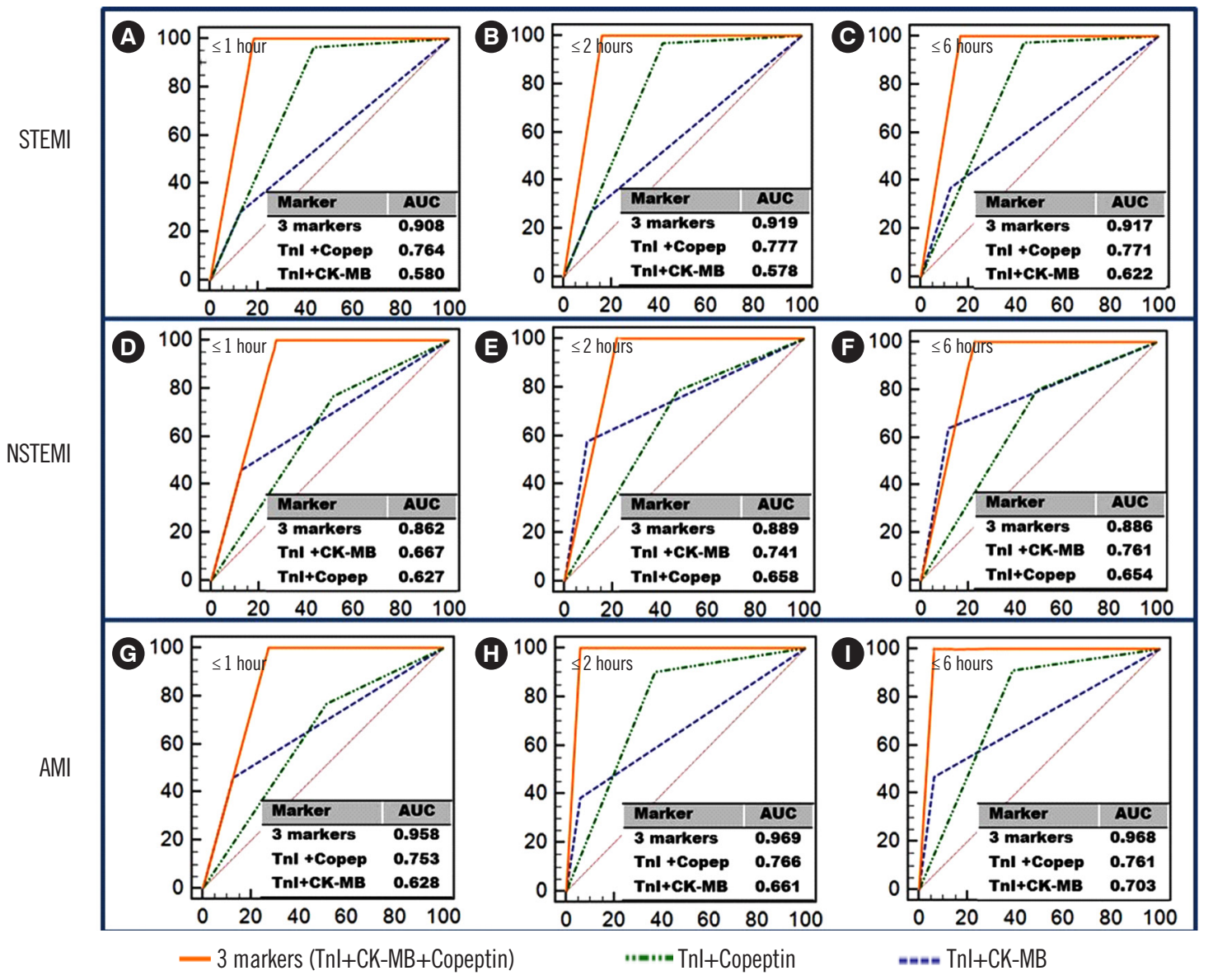

Fig. 2. ROC curves for combined markers according to the time of chest pain onset in $\mathrm{AMI}$ patients. A combination of the three markers showed the highest ROC curves in all patient groups. Copeptin plus Tnl showed better diagnostic performance than Tnl plus CK-MB in the STEMI $[(A)-(C)]$ and AMI $[(G)-(I)]$ groups $(P<0.05)$.

Abbreviations: STEMI, ST elevation myocardial infarction; NSTEMI, non-ST elevation myocardial infarction; AMI, acute myocardial infarction; CK-MB, creatine kinase myocardial band; Tnl, troponin I; copep, copeptin. 
Table 2. Sensitivity, specificity, PPV, and NPV for CK-MB, Tnl, and copeptin according to the time of chest pain onset in AMI patients

\begin{tabular}{|c|c|c|c|c|}
\hline \multirow{2}{*}{ Marker $^{\dagger}$} & & \multicolumn{3}{|c|}{ Chest pain onset (N of AMI patients/total) } \\
\hline & & $\leq 1$ hour (41/147) & $\leq 2$ hours (52/213) & $\leq 6$ hours $(68 / 271)$ \\
\hline \multirow[t]{4}{*}{ CK-MB } & Sen $(95 \% \mathrm{Cl})$ & $31.71(18.08-48.09)$ & $32.69(20.33-47.11)$ & $41.18(29.37-53.77)$ \\
\hline & $\mathrm{Sp}(95 \% \mathrm{Cl})$ & $91.51(84.49-96.04)$ & $93.79(88.87-96.98)$ & $94.09(89.90-96.91)$ \\
\hline & PPV (95\% Cl) & $59.09(40.08-75.72)$ & $62.69(45.38-77.67)$ & $70.00(55.71-81.23)$ \\
\hline & $\mathrm{NPV}(95 \% \mathrm{Cl})$ & $77.60(73.61-81.14)$ & $81.18(78.05-83.96)$ & $82.68(79.60-85.39)$ \\
\hline \multirow[t]{4}{*}{ Tnl } & Sen $(95 \% \mathrm{Cl})$ & $19.51(8.82-34.87)$ & $21.15(11.06-34.70)$ & $32.35(21.51-44.79)$ \\
\hline & $\mathrm{Sp}(95 \% \mathrm{Cl})$ & $99.06(94.86-99.98)$ & $99.38(96.59-99.98)$ & $99.01(96.49-99.88)$ \\
\hline & PPV $(95 \% \mathrm{Cl})$ & $88.89(50.80-98.41)$ & $91.67(59.26-98.81)$ & $91.67(72.64-97.85)$ \\
\hline & NPV $(95 \% \mathrm{Cl})$ & $76.09(73.22-78.74)$ & $79.60(77.21-81.80)$ & $81.38(78.75-83.75)$ \\
\hline \multirow[t]{4}{*}{ Copeptin } & $\operatorname{Sen}(95 \% \mathrm{Cl})$ & $87.80(73.80-95.92)$ & $84.62(71.92-93.12)$ & $79.41(67.88-88.26)$ \\
\hline & $\mathrm{Sp}(95 \% \mathrm{Cl})$ & $60.38(50.41-69.75)$ & $62.73(54.77-70.21)$ & $61.58(54.51-68.30)$ \\
\hline & PPV $(95 \% \mathrm{Cl})$ & $46.15(39.76-52.67)$ & $42.31(36.78-48.03)$ & $40.91(35.90-46.12)$ \\
\hline & $\mathrm{NPV}(95 \% \mathrm{Cl})$ & $92.75(84.73-96.72)$ & $92.66(86.84-96.02)$ & $89.93(84.68-93.51)$ \\
\hline \multirow[t]{4}{*}{ Tnl, Copeptin } & Sen $(95 \% \mathrm{Cl})$ & $90.24(76.87-97.28)$ & $90.38(78.97-96.80)$ & $91.18(81.78-96.69)$ \\
\hline & $\mathrm{Sp}(95 \% \mathrm{Cl})$ & $60.38(50.41-69.75)$ & $62.73(54.77-70.21)$ & $61.08(54.01-67.83)$ \\
\hline & PPV (95\% Cl) & $46.84(40.55-53.22)$ & $43.93(38.62-49.37)$ & $43.97(39.42-48.63)$ \\
\hline & NPV (95\% Cl) & $94.12(86.16-97.63)$ & $95.28(89.70-97.91)$ & 95.38 (90.52-97.81) \\
\hline
\end{tabular}

Abbreviations: CK-MB, creatine kinase myocardial band; Sen, sensitivity; Sp, specificity; PPV, positive predictive value; NPV, negative predictive value.

Table 3. Elevation of cardiac markers according to the time of chest pain onset in AMI patients

\begin{tabular}{|c|c|c|c|c|c|c|c|c|c|}
\hline & \multicolumn{3}{|c|}{ STEMI } & \multicolumn{3}{|c|}{ NSTEMI } & \multicolumn{3}{|c|}{ AMI } \\
\hline & $\begin{array}{l}\leq 1 \text { hour } \\
(28 / 147)\end{array}$ & $\begin{array}{l}\leq 2 \text { hours } \\
(33 / 213)\end{array}$ & $\begin{array}{l}\leq 6 \text { hours } \\
(43 / 271)\end{array}$ & $\begin{array}{l}\leq 1 \text { hour } \\
(13 / 147)\end{array}$ & $\begin{array}{l}\leq 2 \text { hours } \\
(19 / 213)\end{array}$ & $\begin{array}{l}\leq 6 \text { hours } \\
(25 / 271)\end{array}$ & $\begin{array}{l}\leq 1 \text { hour } \\
(41 / 147)\end{array}$ & $\begin{array}{l}\leq 2 \text { hours } \\
(52 / 213)\end{array}$ & $\begin{array}{l}\leq 6 \text { hours } \\
(68 / 271)\end{array}$ \\
\hline CK-MB, N (\%) & $7(25.0)$ & $8(24.2)$ & $14(32.6)$ & $6(46.2)$ & $9(47.4)$ & $14(56.0)$ & $13(31.7)$ & $17(32.7)$ & 28 (41.2) \\
\hline Tnl, N (\%) & $5(17.9)$ & $5(15.2)$ & $12(27.9)$ & $3(23.1)$ & $6(31.6)$ & $10(40.0)$ & $8(19.5)$ & $11(21.2)$ & $22(32.4)$ \\
\hline Copeptin, N (\%) & 26 (92.9) & 31 (93.9) & $38(88.4)$ & $10(76.9)$ & $13(68.4)$ & $16(64.0)$ & $36(87.8)$ & $44(84.6)$ & 54 (79.4) \\
\hline
\end{tabular}

Abbreviations: AMI, acute myocardial infarction; STEMI, ST elevation myocardial infarction; NSTEMI, non-ST elevation myocardial infarction; CK-MB, creatine kinase myocardial band; Tnl, troponin I.

tom onset, even before Tnl elevation (Table 3). In patients at $\leq$ one hour after chest pain onset, only 19.5\% showed Tnl elevation, whereas copeptin was elevated in $87.8 \%$ of AMI patients. In particular, at $\leq$ two hours after chest pain onset, copeptin was elevated in more than $90 \%$ of STEMI patients. Copeptin responded more rapidly after chest pain onset than Tnl or CK-MB (Table 3).

\section{DISCUSSION}

We assessed the diagnostic performance of serum copeptin alone and in combination with CK-MB and/or Tnl in patients with acute chest pain ( $\leq$ six hours) who presented at an ED. Approximately $25.1 \%$ of the patients had $\mathrm{AMI}$, and more than half of our patients (147/271) presented within one hour of chest pain onset. Thus, our cohort was suitable for evaluating the diagnostic performance of copeptin as an early biomarker of AMI.

AMI is a major cause of mortality, and rapid diagnosis of AMI is essential for treatment. Although cardiac troponin has a diagnostic value and is mostly used to diagnose AMI, it remains suboptimal in terms of early risk assessment due to the releasing pathophysiology. In the case of early AMI, repetitive tests for cardiac troponin are needed to achieve prognostic accuracy. The 2014 American Heart Association/American College of Cardiology (AHA/ACC) guidelines also recommend a first measurement at presentation and a second measurement within six hours of arrival. Therefore, a diagnostic maker for AMI that responds earlier than Tnl is needed.

Boeddinghaus, et al. [16] reported that only $6-22 \%$ of pa- 
tients presenting at an ED with suggestive AMI had mild TnT/I elevation at presentation. In the present study, only 19.5\% (STEMI: 17.9\%, NSTEMI: $23.1 \%$ ) of patients who presented within one hour of chest pain onset showed Tnl elevation, whereas serum copeptin levels were elevated in $87.8 \%$ of AMI, $92.9 \%$ of STEMI, and $76.9 \%$ of NSTEMI patients. Our results confirm that Tnl elevation in AMI is delayed for several hours after chest pain onset [17-19]. The early copeptin response was especially prominent in STEMI patients (Table 3, Fig. 1), which concurs with the findings of Reichlin, et al. [17].

The diagnostic value of copeptin as a single biomarker was superior to that of CK-MB or Tnl in AMI and STEMI patients within one hour after chest pain onset (Fig. 1). Although copeptin showed high sensitivity and NPV (sensitivity: 79.41-87.80\%; NPV: 89.9398.17\%), its specificity and PPV were low (specificity: 60.3862.73\%; PPV: 40.91-46.15\%), which hinder its use as a single diagnostic biomarker in AMI. Addition of copeptin to Tnl improved sensitivity and NPV compared with Tnl alone (Table 2). These findings are consistent with the previous findings [20, 21]. ROC curve comparison results showed that a combination of Tnl and copeptin also improved diagnostic performance, specifically at $\leq$ two hours after chest pain onset; thus, this combination might accelerate therapeutic decision-making in patients with suspected AMI. Using Tnl, copeptin, or a combination of two biomarkers may be more efficacious than using CK-MB, especially in STEMI. The CHOPIN trial also showed that copeptin and $\mathrm{Tnl}$ in combination allowed AMI to be ruled out in patients presenting early with suspected ACS [6]. Furthermore, a combination of copeptin and Tnl at presentation has a high NPV to obviate serial analysis beyond three hours of chest pain onset and to facilitate decision making in patients with chest pain in an ED setting [6].

This study is limited by its single-center design and because the blood samples were not obtained serially from the time of symptom onset, which prevented us from examining the relationship between copeptin levels and the time after pain onset in individual patients. Furthermore, we did not perform additional statistical analyses such as the integrated discrimination increment and net reclassification indices.

In summary, copeptin used in combination with Tnl improves the diagnosis of AMI in early presenters because copeptin is different from pathophysiological biomarkers. Addition of copeptin to Tnl improved sensitivity and NPV, and both markers are complementary in early diagnosis of AMI. A Tnl plus copeptin dual marker strategy, rather than a Tnl plus CK-MB strategy, might facilitate "ruling in" and "ruling out" AMI in early presenters in the ED setting.

\section{Authors' Disclosures of Potential Conflicts of Interest}

The authors declare that they have no conflict of interests.

\section{Acknowledgements}

Thermo Fisher Scientific Korea supplied the assay kits and the analyzer. The sponsor had no role in the study design, data collection and analysis, or writing of manuscript.

\section{Author Contributions}

Research conception and design: Ji Hun Jeong, Yiel Hea Seo, Jeong Yeal Ahn, Kyung Hee Kim, Ja Young Seo, Pil Whan Park. Data acquisition: Ji Hun Jeong. Review of patients' clinical information: Ka Yeong Chun, Yong Su Lim. Data analysis and interpretation: Ji Hun Jeong, Pil Whan Park. Statistical analysis: Ji Hun Jeong. Drafting of the manuscript: Ji Hun Jeong, Pil Whan Park. Critical revision of the manuscript: Ji Hun Jeong, Pil Whan Park. Approval of final manuscript: all authors.

\section{ORCID}

Ji Hun Jeong

Yiel Hea Seo

Jeong Yeal Ahn

Kyung Hee Kim

Ja Young Seo

Ka Yeong Chun

Yong Su Lim

Pil Whan Park

\begin{abstract}
https://orcid.org/0000-0002-5586-7889 https://orcid.org/0000-0001-6849-122X https://orcid.org/0000-0001-9842-0748 https://orcid.org/0000-0002-6433-454X https://orcid.org/0000-0002-1894-1365 https://orcid.org/0000-0002-1749-5850 https://orcid.org/0000-0003-4390-4010 https://orcid.org/0000-0002-4955-6240
\end{abstract}

\section{REFERENCES}

1. Makam AN and Nguyen OK. Use of cardiac biomarker testing in the emergency department. JAMA Intern Med 2015;175:67-75.

2. Hajar R. Evolution of myocardial infarction and its biomarkers: a historical perspective. Heart Views 2016;17:167-72.

3. Plebani M, Antonelli G, Zaninotto M. Cardiac biomarkers of acute coronary syndrome: from history to high-sensitive cardiac troponin. Intern Emerg Med 2017;12:143-5.

4. Morgenthaler NG, Struck J, Jochberger S, Dünser MW. Copeptin: clinical use of a new biomarker. Trend Endocrinol Metab 2008;19:43-9.

5. Slagman A, Searle J, Müller C, Möckel M. Temporal release pattern of copeptin and troponin $\mathrm{T}$ in patients with suspected acute coronary syn- 
drome and spontaneous acute myocardial infarction. Clin Chem 2015; 61:1273-82.

6. Maisel A, Mueller C, Neath SX, Christenson RH, Morgenthaler NG, McCord J, et al. Copeptin helps in the early detection of patients with acute myocardial infarction: primary results of the CHOPIN trial (Copeptin Helps in the early detection Of Patients with acute myocardial INfarction). J Am Coll Cardiol 2013;62:150-60.

7. Roffi M, Patrono C, Collet JP, Mueller C, Valgimigli M, Andreotti F, et al. 2015 ESC Guidelines for the management of acute coronary syndromes in patients presenting without persistent ST-segment elevation: Task Force for the Management of Acute Coronary Syndromes in Patients Presenting without Persistent ST-Segment Elevation of the European Society of Cardiology (ESC). Eur Heart J 2016;37:267-315.

8. Reinstadler SJ, Klug G, Feistritzer HJ, Metzler B, Mair J. Copeptin testing in acute myocardial infarction: ready for routine use? Dis Markers 2015;2015.

9. Müller B, Morgenthaler N, Stolz D, Schuetz P, Müller C, Bingisser R, et al. Circulating levels of copeptin, a novel biomarker, in lower respiratory tract infections. Eur J Clin Invest 2007;37:145-52.

10. Morgenthaler NG, Müller B, Struck J, Bergmann A, Redl H, Christ-Crain M. Copeptin, a stable peptide of the arginine vasopressin precursor, is elevated in hemorrhagic and septic shock. Shock 2007;28:219-26.

11. Katan M, Fluri F, Morgenthaler NG, Schuetz P, Zweifel C, Bingisser R, et al. Copeptin: a novel, independent prognostic marker in patients with ischemic stroke. Ann Neurol 2009;66:799-808.

12. Möckel M and Searle J. Copeptin—marker of acute myocardial infarction. Curr Atheroscler Rep 2014;16:1-8.

13. Morgenthaler NG, Struck J, Alonso C, Bergmann A. Assay for the measurement of copeptin, a stable peptide derived from the precursor of vasopressin. Clin Chem 2006;52:112-9.
14. Marston NA, Shah KS, Mueller C, Neath SX, Christenson RH, McCord J, et al. Serial sampling of copeptin levels improves diagnosis and risk stratification in patients presenting with chest pain: results from the CHOPIN trial. Emerg Med J 2016;33:23-9.

15. Lipinski MJ, Escárcega RO, D’Ascenzo F, Magalhães MA, Baker NC, Torguson $\mathrm{R}$, et al. A systematic review and collaborative meta-analysis to determine the incremental value of copeptin for rapid rule-out of acute myocardial infarction. Am J Cardiol 2014;113:1581-91.

16. Boeddinghaus J, Reichlin T, Nestelberger T, Twerenbold R, Meili Y, Wildi $\mathrm{K}$, et al. Early diagnosis of acute myocardial infarction in patients with mild elevations of cardiac troponin. Clin Res Cardiol 2017;106:457-67.

17. Reichlin T, Hochholzer W, Stelzig C, Laule K, Freidank H, Morgenthaler NG, et al. Incremental value of copeptin for rapid rule out of acute myocardial infarction. J Am Coll Cardiol 2009;54:60-8.

18. MacRae AR, Kavsak PA, Lustig V, Bhargava R, Vandersluis R, Palomak $\mathrm{GE}$, et al. Assessing the requirement for the 6-hour interval between specimens in the American Heart Association Classification of Myocardial Infarction in Epidemiology and Clinical Research Studies. Clin Chem 2006;52:812-8.

19. Melanson SE, Morrow DA, Jarolim P. Earlier detection of myocardial injury in a preliminary evaluation using a new troponin I assay with improved sensitivity. Am J Clin Pathol 2007;128:282-6.

20. Shin H, Jang BH, Lim TH, Lee J, Kim W, Cho Y, et al. Diagnostic accuracy of adding copeptin to cardiac troponin for non-ST-elevation myocardial infarction: a systematic review and meta-analysis. PloS One 2018; 13:e0200379.

21. Mueller C, Möckel M, Giannitsis E, Huber K, Mair J, Plebani M, et al. Use of copeptin for rapid rule-out of acute myocardial infarction. Eur Heart J Acute Cardiovasc Care 2017:2048872617710791. 